\section{Folgen der EBM-Weiterentwicklung für das Fachgebiet Radiologie}

Am 01.04.2020 trat der neue, reformierte Einheitliche Bewertungsmaßstab (EBM) in Kraft. Nach fast acht Jahren der Überarbeitungen und Abstimmungen erfolgte die Verabschiedung des neuen EBM durch den Bewertungsausschuss am 11.12.2019. Eine Reform als planvolle Umgestaltung des ambulanten Vergütungssystems in der gesetzlichen Krankenversicherung stellt der neue EBM sicherlich nicht dar. Auf der Grundlage der $§ 87$ Abs. 2 des fünften Buch Sozialgesetzbuch (SGB V) erfolgte die seit vielen Jahren ausstehende Überprüfung und Neubewertung der Gebührenordnungspositionen (GOP). Der Ansatz des EBM war und ist es, die verschiedenen Leistungen in der ambulanten Versorgung von gesetzlich krankenversicherten Patienten in ein wertmäßiges Verhältnis zueinander zu setzen. Die Aufwertung der sprechenden Medizin ist zwar eine gesundheitspolitisch zulässige Forderung und kann vom Gesetzgeber gefordert werden, bringt aber das Gefüge des wertmäßigen Verhältnisses der ambulanten Leistungen durcheinander, wenn diese Aufwertung über Anpassungen des EBM und damit das gesamte Vergütungssystem in der ambulanten Versorgung gesetzlich krankenversicherter Patienten erfolgt.

\section{Wertmäßiges Verhältnis der Leistungen zueinander}

Seit eh und je lautet die Kernformulierung in $\S 87$ Abs. 2 Satz 1 SGB V:

„Der einheitliche Bewertungsmaßstab bestimmt den Inhalt der abrechnungsfähigen Leistungen und ihr wertmäßiges, in Punkten ausgedrücktes Verhältnis zueinander; soweit möglich, sind die Leistungen mit Angaben für den zur Leistungserbringung erforderlichen Zeitaufwand des Vertragsarztes zu versehen [...].“

Das Gesetz sah seit der Einführung des EBM vor, dass der Bewertungsmaßstab in bestimmten Zeitabständen daraufhin zu überprüfen sei, ob die enthaltenen Leistungsbeschreibungen und ihre Bewertungen noch dem aktuellen Stand der medizinischen Wissenschaft und Technik sowie dem
Erfordernis der Rationalisierung im Rahmen wirtschaftlicher Leistungserbringung entsprächen. Bei der Bewertung der Leistungen sollte insbesondere der Aspekt der wirtschaftlichen Nutzung der bei der Erbringung von Leistungen eingesetzten medizinischtechnischen Geräte berücksichtigt werden. Nach §87 Abs. 2 Satz 3 SGB V gilt, dass im EBM die Bewertung der Leistungen und die Überprüfung der wirtschaftlichen Aspekte, insbesondere bei medizinisch-technischen Geräten, unter Berücksichtigung der Besonderheiten der betroffenen Arztgruppen auf in bestimmten Zeitabständen zu aktualisierender betriebswirtschaftlicher Basis durchzuführen sind. Die „bestimmten Zeitabstände" nach § 87 Abs. 2 Sätze 1 und 3 SGB V hat der Gesetzgeber indes nie definiert und so lag es an der Kassenärztlichen Bundesvereinigung (KBV) und dem Spitzenverband Bund der Krankenkassen (GKV-Spitzenverband) diese Überprüfung im Bewertungsausschuss vorzunehmen.

Mit dem Terminservicestellen- und Versorgungsgesetz (TSVG), das am 11.05.2019 in Kraft trat, schrieb der Gesetzgeber der KBV und dem GKV-Spitzenverband vor, dass die Bewertung der Leistungen und die Überprüfung der wirtschaftlichen Aspekte, insbesondere bei medizinisch-technischen Geräten, bis zum 29.02.2020 zu erfolgen habe. Mit dieser gesetzlichen Fristsetzung für die Aktualisierung des einheitlichen Bewertungsmaßstabs und der Frist zur Vorlage eines Aktualisierungskonzeptes entstand, wie von dem Gesetzgeber ausdrücklich gewollt, für den Bewertungsausschuss die Notwendigkeit, die erforderlichen Arbeiten zur Weiterentwicklung des EBM unverzüglich aufzunehmen, um eine fristgerechte Beschlussfassung sicherzustellen.

Der Gesetzgeber gab in der Begründung des TSVG an, dass mit der Neufassung des $\S 87$ Abs. 2 Satz 3 SGB V Rationalisierungsreserven bei dem Einsatz von medizinisch-technischen Geräten im einheitlichen Bewertungsmaßstab für ärztliche Leistungen zugunsten von Verbesserungen von zuwendungsorientierten ärztlichen Leistungen („sprechende Medizin“) genutzt werden sollten. Rationalisierungsreserven ergäben sich aufgrund des

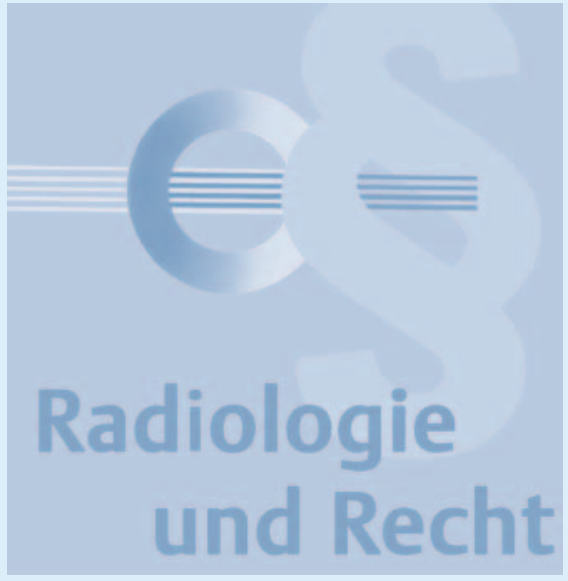

technischen Fortschritts durch Automatisierung und Digitalisierung, weiterhin aufgrund einer veralteten betriebswirtschaftlichen Kalkulation und einer veralteten Datengrundlage der Bewertungen einschließlich der vorgesehenen Zeitaufwände. Der Gesetzgeber ließ es nach der Begründung des TSVG, ohne dies aber im SGB V selbst zu verankern, zu, dass der Bewertungsausschuss eine Konzentrierung auf Arztgruppen oder Leistungen mit einem hohen technischen Leistungsanteil z. B. Strahlentherapie und Nuklearmedizin, Labor, Radiologie oder Humangenetik vornimmt. Die Bewertung der technischen Leistungen solle so festgelegt werden, dass die punktzahlmäßige Bewertung der in einem bestimmten Zeitraum erbrachten Leistungen oder Leistungsanteile ab einem bestimmten Schwellenwert mit zunehmender Menge sinke, wie es in der Begründung des TSVG wie auch in $\S 87$ Abs. 2 Satz 7 SGB V vorgesehen ist.

Die Konzentration auf die Leistungen der Arztgruppen Radiologie, Nuklearmedizin oder Strahlentherapie fand letztlich nicht statt, sondern es erfolgte eine umfassende Überarbeitung der Leistungsbewertungen.

Eine generelle Änderung, die über alle Arztgruppen erfolgte, ist die Erhöhung eines kalkulatorischen Arztlohnes. Seit dem Beschluss des Erweiterten Bewertungsausschusses in seiner 5 . Sitzung vom 11 . und 12. Oktober 2007 betrug der kalkulatorische Arztlohn 105.571,80 Euro. Den im Standardbewertungssystem ab dem 01.04.2020 verwendeten kalkulatorischen Arztlohn setze der Bewertungsausschuss im Beschluss vom 11.12.2019 in seiner 455. Sitzung in Teil E mit 117.060,00 Euro fest. 


\section{Änderungen des EBM im Einzelnen}

Aus der nachfolgenden Darstellung (prozentuale Veränderungen der Euro- und Punktwerte durch den Verfasser ermittelt) gehen die Bewertungen der einzelnen wesentlichen radiologischen Leistungen bis zum 31.03.2020 und ab dem 01.04.2020 im Vergleich hervor.

EBM bis 31.03.2020

GOP
Euro
EBM ab 01.04.2020

Euro
Euro-Veränderung in \%
Punkte

Punkteveränderung

in \%

\section{Konsiliarpauschale}

\begin{tabular}{|c|c|c|c|c|c|c|}
\hline 24210 & 5,82 & 53 & 8,02 & $37,80 \%$ & 73 & $37,74 \%$ \\
\hline 24211 & 4,83 & 44 & 6,7 & $38,72 \%$ & 61 & $38,64 \%$ \\
\hline 24212 & 5,82 & 53 & 8,02 & $37,80 \%$ & 73 & $37,74 \%$ \\
\hline 24228 & KV-individuell & & KV-individuell & & & \\
\hline \multicolumn{7}{|c|}{ Röntgen } \\
\hline 34210 & 11,65 & 106 & 11,32 & $-2,83 \%$ & 103 & $-2,83 \%$ \\
\hline 34211 & 8,13 & 74 & 7,8 & $-4,06 \%$ & 71 & $-4,05 \%$ \\
\hline 34212 & 11,65 & 106 & 11,21 & $-3,78 \%$ & 102 & $-3,77 \%$ \\
\hline 34220 & 10,44 & 95 & 10 & $-4,21 \%$ & 91 & $-4,21 \%$ \\
\hline 34221 & 16,70 & 152 & 15,38 & $-7,90 \%$ & 140 & $-7,89 \%$ \\
\hline 34222 & 20,55 & 187 & 18,02 & $-12,31 \%$ & 164 & $-12,30 \%$ \\
\hline 34223 & 82,73 & 753 & 77,13 & $-6,77 \%$ & 702 & $-6,77 \%$ \\
\hline 34230 & 8,57 & 78 & 8,13 & $-5,13 \%$ & 74 & $-5,13 \%$ \\
\hline 34231 & 15,49 & 141 & 15,05 & $-2,84 \%$ & 137 & $-2,84 \%$ \\
\hline 34232 & 11,65 & 106 & 10,88 & $-6,61 \%$ & 99 & $-6,60 \%$ \\
\hline 34233 & 11,65 & 106 & 10,88 & $-6,61 \%$ & 99 & $-6,60 \%$ \\
\hline 34234 & 8,13 & 74 & 7,8 & $-4,06 \%$ & 71 & $-4,05 \%$ \\
\hline 34235 & 72,30 & 658 & 67,13 & $-7,15 \%$ & 611 & $-7,14 \%$ \\
\hline 34236 & 59,99 & 546 & 56,47 & $-5,87 \%$ & 514 & $-5,86 \%$ \\
\hline 34237 & 15,49 & 141 & 16,92 & $9,23 \%$ & 154 & $9,22 \%$ \\
\hline 34238 & 11,65 & 106 & 10,88 & $-6,61 \%$ & 99 & $-6,60 \%$ \\
\hline 34240 & 9,34 & 85 & 9,01 & $-3,53 \%$ & 82 & $-3,53 \%$ \\
\hline 34241 & 16,70 & 152 & 16,04 & $-3,95 \%$ & 146 & $-3,95 \%$ \\
\hline 34242 & 32,41 & 295 & 29,23 & $-9,81 \%$ & 266 & $-9,83 \%$ \\
\hline 34243 & 10,44 & 95 & 10,22 & $-2,11 \%$ & 93 & $-2,11 \%$ \\
\hline 34244 & 15,49 & 141 & 15,49 & $0,00 \%$ & 141 & $0,00 \%$ \\
\hline 34245 & 11,65 & 106 & 11,65 & $0,00 \%$ & 106 & $0,00 \%$ \\
\hline 34246 & 33,18 & 302 & 31,75 & $-4,31 \%$ & 289 & $-4,30 \%$ \\
\hline 34247 & 52,08 & 474 & 49,22 & $-5,49 \%$ & 448 & $-5,49 \%$ \\
\hline 34248 & 116,02 & 1056 & 113,94 & $-1,79 \%$ & 1037 & $-1,80 \%$ \\
\hline 34250 & 46,04 & 419 & 43,73 & $-5,02 \%$ & 398 & $-5,01 \%$ \\
\hline 34251 & 97,68 & 889 & 96,58 & $-1,13 \%$ & 879 & $-1,12 \%$ \\
\hline 34252 & 83,94 & 764 & 81,3 & $-3,15 \%$ & 740 & $-3,14 \%$ \\
\hline 34255 & 46,59 & 424 & 48,01 & $3,05 \%$ & 437 & $3,07 \%$ \\
\hline 34256 & 63,51 & 578 & 60,32 & $-5,02 \%$ & 549 & $-5,02 \%$ \\
\hline 34257 & 97,13 & 884 & 92,84 & $-4,42 \%$ & 845 & $-4,41 \%$ \\
\hline 34260 & 41,42 & 377 & 39,88 & $-3,72 \%$ & 363 & $-3,71 \%$ \\
\hline
\end{tabular}




\begin{tabular}{|c|c|c|c|c|c|c|}
\hline \multirow[b]{2}{*}{ GOP } & \multicolumn{2}{|c|}{ EBM bis 31.03 .2020} & \multicolumn{4}{|c|}{ EBM ab 01.04.2020 } \\
\hline & Euro & Punkte & Euro & $\begin{array}{l}\text { Euro-Veränderung } \\
\text { in \% }\end{array}$ & Punkte & $\begin{array}{l}\text { Punkteveränderung } \\
\text { in \% }\end{array}$ \\
\hline 34270 & 29,12 & 265 & 30,1 & $3,37 \%$ & 274 & $3,40 \%$ \\
\hline 34271 & 91,52 & 833 & 95,48 & $4,33 \%$ & 869 & $4,32 \%$ \\
\hline 34272 & 26,81 & 244 & 29,34 & $9,44 \%$ & 267 & $9,43 \%$ \\
\hline 34273 & 9,12 & 83 & 10,77 & $18,09 \%$ & 98 & $18,07 \%$ \\
\hline 34274 & 29,88 & 272 & 29,88 & $0,00 \%$ & 272 & $0,00 \%$ \\
\hline 34275 & 21,75 & 198 & 23,4 & $7,59 \%$ & 213 & $7,58 \%$ \\
\hline 34280 & 10,44 & 95 & 10,44 & $0,00 \%$ & 95 & $0,00 \%$ \\
\hline 34281 & 6,59 & 60 & 6,81 & $3,34 \%$ & 62 & $3,33 \%$ \\
\hline 34282 & 43,95 & 400 & 40,87 & $-7,01 \%$ & 372 & $-7,00 \%$ \\
\hline 34283 & 170,52 & 1552 & 170,52 & $0,00 \%$ & 1552 & $0,00 \%$ \\
\hline 34284 & 107,45 & 978 & 107,89 & $0,41 \%$ & 982 & $0,41 \%$ \\
\hline 34285 & 52,63 & 479 & 52,41 & $-0,42 \%$ & 477 & $-0,42 \%$ \\
\hline 34286 & 243,58 & 2217 & 244,02 & $0,18 \%$ & 2221 & $0,18 \%$ \\
\hline 34287 & 13,84 & 126 & 13,73 & $-0,79 \%$ & 125 & $-0,79 \%$ \\
\hline 34290 & 132,50 & 1206 & 154,26 & $16,42 \%$ & 1404 & $16,42 \%$ \\
\hline 34291 & 354,55 & 3227 & 348,84 & $-1,61 \%$ & 3175 & $-1,61 \%$ \\
\hline 34292 & 419,60 & 3819 & 417,4 & $-0,52 \%$ & 3799 & $-0,52 \%$ \\
\hline 34293 & 85,48 & 778 & 74,71 & $-12,60 \%$ & 680 & $-12,60 \%$ \\
\hline 34294 & 44,28 & 403 & 38,78 & $-12,42 \%$ & 353 & $-12,41 \%$ \\
\hline 34295 & 8,90 & 81 & 10,44 & $17,30 \%$ & 95 & $17,28 \%$ \\
\hline 34296 & 89,33 & 813 & 85,7 & $-4,06 \%$ & 780 & $-4,06 \%$ \\
\hline 34297 & 105,48 & 960 & 99,21 & $-5,94 \%$ & 903 & $-5,94 \%$ \\
\hline 34298 & 107,67 & 980 & 107,67 & $0,00 \%$ & 980 & $0,00 \%$ \\
\hline \multicolumn{7}{|l|}{ CT } \\
\hline 34310 & 67,46 & 614 & 58,67 & $-13,03 \%$ & 534 & $-13,03 \%$ \\
\hline 34311 & 81,63 & 743 & 72,73 & $-10,90 \%$ & 662 & $-10,90 \%$ \\
\hline 34312 & 46,81 & 426 & 43,29 & $-7,52 \%$ & 394 & $-7,51 \%$ \\
\hline 34320 & 81,74 & 744 & 71,42 & $-12,63 \%$ & 650 & $-12,63 \%$ \\
\hline 34321 & 69,33 & 631 & 61,64 & $-11,09 \%$ & 561 & $-11,09 \%$ \\
\hline 34322 & 84,49 & 769 & 74,38 & $-11,97 \%$ & 677 & $-11,96 \%$ \\
\hline 34330 & 72,51 & 660 & 64,38 & $-11,21 \%$ & 586 & $-11,21 \%$ \\
\hline 34340 & 72,84 & 663 & 63,84 & $-12,36 \%$ & 581 & $-12,37 \%$ \\
\hline 34341 & 89,98 & 819 & 79,55 & $-11,59 \%$ & 724 & $-11,60 \%$ \\
\hline 34342 & 72,84 & 663 & 63,84 & $-12,36 \%$ & 581 & $-12,37 \%$ \\
\hline 34343 & 54,06 & 492 & 47,35 & $-12,41 \%$ & 431 & $-12,40 \%$ \\
\hline 34344 & 55,92 & 509 & 51,2 & $-8,44 \%$ & 466 & $-8,45 \%$ \\
\hline 34345 & 25,05 & 228 & 23,73 & $-5,27 \%$ & 216 & $-5,26 \%$ \\
\hline 34350 & 62,52 & 589 & 54,94 & $-12,12 \%$ & 500 & $-15,11 \%$ \\
\hline 34351 & 62,52 & 569 & 54,94 & $-12,12 \%$ & 500 & $-12,13 \%$ \\
\hline \multicolumn{7}{|c|}{ CT-Bestrahlungsplanung } \\
\hline 34360 & 42,52 & 387 & 38,89 & $-8,54 \%$ & 354 & $-8,53 \%$ \\
\hline
\end{tabular}


EBM bis 31.03.2020

GOP
Euro
EBM ab 01.04.2020

Euro
Euro-Veränderung in \%
Punkte
Punkteveränderung in \%

\begin{tabular}{|c|c|c|c|c|c|c|}
\hline \multicolumn{7}{|l|}{ MRT } \\
\hline 34410 & 133,27 & 1213 & 115,69 & $-13,19 \%$ & 1053 & $-13,19 \%$ \\
\hline 34411 & 133,27 & 1213 & 115,69 & $-13,19 \%$ & 1053 & $-13,19 \%$ \\
\hline 34420 & 133,27 & 1213 & 115,69 & $-13,19 \%$ & 1053 & $-13,19 \%$ \\
\hline 34421 & 133,27 & 1213 & 115,69 & $-13,19 \%$ & 1053 & $-13,19 \%$ \\
\hline 34422 & 133,27 & 1213 & 115,69 & $-13,19 \%$ & 1053 & $-13,19 \%$ \\
\hline 34430 & 133,27 & 1213 & 115,69 & $-13,19 \%$ & 1053 & $-13,19 \%$ \\
\hline 34431 & 240,95 & 2193 & 115,69 & $-51,99 \%$ & 1053 & $-51,98 \%$ \\
\hline 34440 & 133,27 & 1213 & 115,69 & $-13,19 \%$ & 1053 & $-13,19 \%$ \\
\hline 34441 & 133,27 & 1213 & 115,69 & $-13,19 \%$ & 1053 & $-13,19 \%$ \\
\hline 34442 & 133,27 & 1213 & 115,69 & $-13,19 \%$ & 1053 & $-13,19 \%$ \\
\hline 34450 & 133,27 & 1213 & 115,69 & $-13,19 \%$ & 1053 & $-13,19 \%$ \\
\hline 34451 & 133,27 & 1213 & 115,69 & $-13,19 \%$ & 1053 & $-13,19 \%$ \\
\hline 34452 & 49,00 & 446 & 41,75 & $-14,80 \%$ & 380 & $-14,80 \%$ \\
\hline 34460 & 91,74 & 835 & 74,38 & $-18,92 \%$ & 677 & $-18,92 \%$ \\
\hline \multicolumn{7}{|c|}{ MRT-Angiografie } \\
\hline 34470 & 87,57 & 797 & 76,03 & $-13,18 \%$ & 692 & $-13,17 \%$ \\
\hline 34475 & 116,35 & 1059 & 100,97 & $-13,22 \%$ & 919 & $-13,22 \%$ \\
\hline 34480 & 116,35 & 1059 & 100,97 & $-13,22 \%$ & 919 & $-13,22 \%$ \\
\hline 34485 & 116,35 & 1059 & 100,97 & $-13,22 \%$ & 919 & $-13,22 \%$ \\
\hline 34486 & 116,35 & 1059 & 100,97 & $-13,22 \%$ & 919 & $-13,22 \%$ \\
\hline 34489 & 235,15 & 2122 & 202,38 & $-13,94 \%$ & 1842 & $-13,20 \%$ \\
\hline 34490 & 116,35 & 1059 & 100,97 & $-13,22 \%$ & 919 & $-13,22 \%$ \\
\hline 34492 & 53,62 & 488 & 45,71 & $-14,75 \%$ & 416 & $-14,75 \%$ \\
\hline \multicolumn{7}{|c|}{ Durchleuchtungs- oder CT-gestützte Intervention } \\
\hline 34500 & 73,83 & 672 & 73,83 & $0,00 \%$ & 672 & $0,00 \%$ \\
\hline 34501 & 98,33 & 895 & 98,33 & $0,00 \%$ & 895 & $0,00 \%$ \\
\hline 34503 & 75,59 & 688 & 73,28 & $-3,06 \%$ & 667 & $-3,05 \%$ \\
\hline 34504 & 108,44 & 987 & 106,36 & $-1,92 \%$ & 968 & $-1,93 \%$ \\
\hline 34505 & 108,44 & 987 & 106,36 & $-1,92 \%$ & 968 & $-1,93 \%$ \\
\hline \multicolumn{7}{|c|}{ Osteodensitometrie } \\
\hline 34600 & 29,45 & 268 & 29,45 & $0,00 \%$ & 268 & $0,00 \%$ \\
\hline 34601 & 29,45 & 268 & 26,45 & $-10,19 \%$ & 268 & $0,00 \%$ \\
\hline
\end{tabular}

Die prozentualen Änderungen der Eurobeträge fallen geringer aus als die prozentualen Änderungen der Punkte. Die geringere Änderung der Eurobeträge geht auf eine Erhöhung des Orientierungspunktwertes zum 01.04.2020 zurück. Daher sind die Auswirkungen der EBM-Reform in Bezirken von Kassenärztlichen Vereinigungen etwas niedriger, wenn diese die Leistungsanforderung in Euro und nicht in Punkten bemessen.
Umsetzung in den Honorarverteilungsmaßstäben der KVen

Letztlich wirkt sich der EBM nur mittelbar auf das Honorar der Radiologen aus. Entscheidend und von unmittelbarer Auswirkung auf das Honorar sind die Honorarverteilungsmaßstäbe der einzelnen Kassenärztlichen Vereinigungen. Seit dem 01.01.2012 hatte der Gesetzgeber die frühere im Wesentlichen bundesweit einheitliche Systematik der Regelleistungsvolumina und qualifikationsgebundenen Zusatzvolumina aufgegeben und den Kassenärztlichen Vereinigungen größere Freiheiten bei der Gestaltung der Honorarverteilung eingeräumt. Seitdem entwickelt sich die Honorarverteilung in den einzelnen Bezirken der Kassenärztlichen Vereinigungen unterschiedlich und die verschiedenen Ansätze konkurrieren nicht miteinander. Soweit 
ersichtlich ist, ließ es die Sozialgerichtsbarkeit bisher nie zu, dass ein Vertragsarzt die Ungleichbehandlung mit einem Vertragsarzt in einem anderen Bezirk einer Kassenärztlichen Vereinigung rügen konnte. Es handle sich dabei um zwei verschiedene Gruppen, die solche Unterschiede aufwiesen, dass sie nicht miteinander verglichen werden könnten. Die verschiedenen Honorarverteilungsmaßstäbe haben nicht nur Folgen für die Entwicklung der Vergütung in der Radiologie, sondern zugleich auf die Rechtsprechung. Ob eine Entscheidung, die zu der Honorarverteilung in einem anderen Bezirk einer Kassenärztlichen Vereinigung erging, auf die eigene Honorarsituation eines Radiologen zu übertragen ist, lässt sich daher aus dem Stegreif nicht mehr beantworten.

In den meisten (Honorar-)Verteilungsmaßstäben setzen die Kassenärztlichen Vereinigungen die EBM-Reform derart um, dass eine fiktive Neuberechnung der Abrechnung des Quartals 2/2019 auf der Grundlage der neuen EBM-Werte erfolgt und diese fiktive Abrechnung Grundlage für die Budgetzuweisung des Quartals 2/2020 ist. Entsprechend erfolgt die Berechnung der Budgets für die späteren Quartale 3/2020 bis 1/2021. Soweit die (Honorar-)Verteilungsmaßstäbe keine Budgets vorsehen, ist darauf zu achten, dass die Verteilung des für die fachärztliche Vergütung zur Verfügung und daneben nicht das konkret für die Arztgruppe der Radiologen zur Vergütung stehende Arztgruppenbudget in einem entsprechenden Verfahren bereinigt wird. In beiden Fällen erfolgt ein Eingriff in den im Vorjahr erarbeiteten Besitzstand. Ob dieser zu schützen ist oder ob die Regelungen über den EBM und die Honorarverteilung eine ausreichende Rechtsgrundlage für diesen Eingriff bieten, wird die Sozialgerichtsbarkeit und letztlich das Bundesverfassungsgericht zu klären haben.

\section{Fazit}

Der ab dem 01.04.2020 geltende EBM reduziert im Wesentlichen die bisherige Wertigkeit der Leistungen der Radiologen. Der Bewertungsausschuss hat dabei Wünsche des Gesetzgebers aus der Gesetzesbegründung Folge geleistet, obwohl aus $\S 87$ Abs. 2 SGB $V$ die Forderung an den Bewertungsausschuss allein darin bestand, medizinisch-technische Leistungen neu zu bewerten. Sprachlich handelte es dabei um einen neutralen Bewertungsansatz. Gegen einen offenen und über alle Arztgruppen gerechten Bewertungsansatz zur Neubewertung dürfte nichts einzuwenden sein. Die wertmäßigen Verhältnisse der Leistungen des EBM zueinander waren bis zum 31.03.2020 neutral und die Förderung von bestimmten Leistungen erfolgte über die Honorarverteilung nach den Verteilungsmaßstäben der Kassenärztlichen Vereinigungen. Seit dem 01.04.2020 entspricht der EBM nicht mehr diesem neutralen wertmäßigen Ansatz. So heißt es in einer Erklärung der KBV vom 23.01.2020 (KBV, aufrufbar unter: https:// www.kbv.de/html/1150_43 855.php, letzter Zugriff 30.03.2020):

"Mit der Anhebung der Bewertung von Gesprächsleistungen soll die sprechende Medizin gefördert werden. Dies war ein Auftrag des Gesetzgebers an die Verhandlungspartner der EBM-Weiterentwicklung. Gleichzeitig sollten die technischen Leistungen überprüft und die Bewertung abgesenkt werden. Denn die Reform des EBM muss ausgabenneutral erfolgen, so die Vorgabe.“

Nicht nur, dass dieser Auftrag allenfalls den Gründen des TSVG aber nicht dem SGB V zu entnehmen ist, erscheint der EBM als vollständig ungeeignet eine Ausgabenneutralität zu gewährleisten. Entscheidend war tat- sächlich schon immer die Honorarverteilung und kaum ein Radiologie dürfte innerhalb der morbiditätsbedingten Gesamtvergütung die Erfahrung gemacht haben, jemals den vorgesehenen Eurowert einer Leistung nach dem EBM erhalten zu haben. Daher wirkt sich die EBM-Reform insgesamt außerordentlich nachteilig aus, weil das tatsächlich in der Vergangenheit für eine einzelne Leistung erreichte Honorar bereits dem EBM-Wert nicht entsprach, weil die Arztgruppenbudgets nicht den tatsächlichen Leistungsanforderungen angepasst worden waren und nunmehr fast durchgängig eine Reduzierung des Arztgruppenbudgets aufgrund einer fiktiven, nach neunen EBM-Werten reduzierten Leistungsanforderung erfolgt. Nach der zitierten Aussage der KBV bedarf es dieses Schrittes nicht, weil die Ausgabenneutralität bereits durch den EBM erfolgte. Warum sollte dann aber eine fiktive Neuberechnung durch den Verteilungsmaßstab erfolgen?

René T. Steinhäuser

Rechtsanwalt

Rechtsanwälte Wigge

Großer Burstah 42

20457 Hamburg

Telefon: (040) 3398 705-90

Telefax: (040) 3398 705-99

E-Mail: kanzlei@ra-wigge.de

www.ra-wigge.de 